\title{
Synchronization and Lag Synchronization of Hyperchaotic Memristor-Based Chua's Circuits
}

\author{
Junjian Huang, ${ }^{1,2}$ Chuandong Li, ${ }^{1,3}$ Tingwen Huang, ${ }^{4}$ Hui Wang, and Xin Wang ${ }^{3}$ \\ ${ }^{1}$ College of Computer Science, Chongqing University, Chongqing 400030, China \\ ${ }^{2}$ Department of Computer Science, Chongqing Education College, Chongqing 400067, China \\ ${ }^{3}$ College of Electronic and Information Science, Southwest University, Chongqing 400715, China \\ ${ }^{4}$ Texas A\&M University at Qatar, P.O. BOX 23874, Doha, Qatar \\ ${ }^{5}$ School of Mathematics and Statistics, Chongqing Normal University, Chongqing 400047, China
}

Correspondence should be addressed to Junjian Huang; hmomu@sina.com and Chuandong Li; licd@cqu.edu.cn

Received 16 July 2014; Accepted 1 September 2014; Published 30 September 2014

Academic Editor: Ramachandran Raja

Copyright (C) 2014 Junjian Huang et al. This is an open access article distributed under the Creative Commons Attribution License, which permits unrestricted use, distribution, and reproduction in any medium, provided the original work is properly cited.

\begin{abstract}
A memristor-based five-dimensional (5D) hyperchaotic Chua's circuit is proposed. Based on the Lyapunov stability theorem, the controllers are designed to realize the synchronization and lag synchronization between the hyperchaotic memristor-based Chua's circuits under different initial values, respectively. Numerical simulations are also presented to show the effectiveness and feasibility of the theoretical results.
\end{abstract}

\section{Introduction}

The fourth fundamental circuit element included along with the resistor, capacitor, and inductor, called the memristor, was first postulated by Chua in 1971 [1]. Until 2008, the HewlettPackard (HP) research team announced that they had realized a prototype of memristor based on nanotechnology [2]. Many researchers focus on the memristor due to its potential applications in programmable logic, signal processing, neural networks, control systems, reconfigurable computing, braincomputer interfaces, and so on [3-9].

Itoh and Muthuswamy presented a fourth-order memristor based on Chua's oscillator by replacing Chua's diode with an active two-terminal circuit consisting of a conductance and a flux-controlled memristor and observed rich nonlinear dynamic behavior in such system $[10,11]$. Bao et al. investigated the initial state dependent dynamical behaviors of the memristor-based chaotic circuit [12, 13]. In [14], a memristor with cubic nonlinear characteristics is employed in the modified canonical Chua circuit. In their work, a systematic study of hyperchaotic behavior in the circuit is performed. Hyperchaotic systems are being developed for applications in secure communications. It is important that memristive hyperchaotic systems are developed for implementation in coming generations of memristor-based devices. Novel dynamical behaviors of the memristor chaotic oscillator system are heavily dependent on the initial state of the memristor except for the circuit parameters. Namely, it is different from the traditional chaotic systems that the memory of initial state of the memristor is very important in producing complicated transient transition dynamics. The chaotic memristor-based oscillator system will enable the production of more complex and unpredictable time domain signals, which may result in applications for secure communications and encryption.

Many works have been done about the stabilization and synchronization of memristor-based systems [15-19]. Zhang et al. presented theoretical results on the global exponential periodicity and stability of a class of memristor-based recurrent neural networks with multiple delays [15]. Wu et al. formulated and investigated a class of memristor-based recurrent neural networks [18]. In this paper, by replacing 


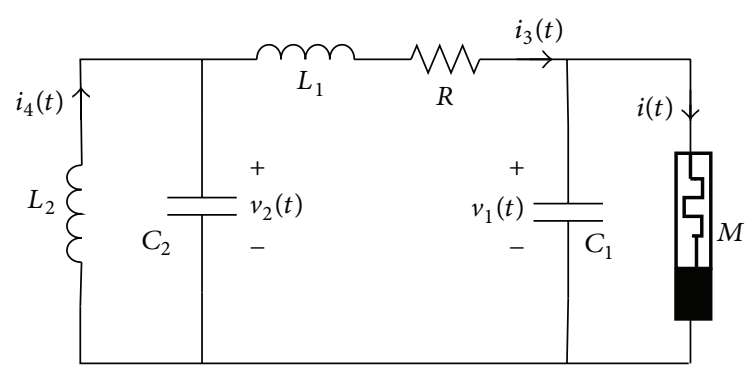

FIgURE 1: The hyperchaotic circuit based on memristor [13].

Chua's diode with a flux-controlled memristor circuit, a memristor-based five-dimensional (5D) hyperchaotic circuit is derived from four-dimensional Chua's oscillator. Based on Lyapunov stability theory, we will study synchronization and lag synchronization of memristor-based hyperchaotic circuits. Hyperchaotic circuits are being developed for applications in secure communications $[20,21]$. It is important that memristive hyperchaotic circuits be developed for implementation in coming generations of memristor-based devices. On the other hand, it has been shown that the complete synchronization of chaos is practically impossible for the finite speed of signals. Chaotic lag synchronization appears as a coincidence of shift-in-time states of interactive systems. It is just synchronization lag that makes lag synchronization practically available. So, in many cases, it is more reasonable to require the slave system to synchronize the master system with a time-delay. Thus, it is of great importance to study lag synchronization. This paper will study synchronization and lag synchronization between the hyperchaotic memristor-based Chua circuits.

The rest of the paper is organized as follows. In Section 2, a memristor-based $5 \mathrm{D}$ chaotic system is introduced. In Sections 3 and 4, using feedback control method, general convergence criterion for synchronization and lag synchronization of memristor-based chaotic hyperchaotic system is established. Numerical simulation results are given to show the effectiveness of the theoretical results. Conclusions are finally drawn in Section 5.

\section{Problem Formulation and Preliminaries}

Referring to [13], by replacing Chua's diode with an active flux-controlled memristor circuit, a memristor-based fivedimensional chaotic circuit is derived from four-order Chua's oscillator, as shown in Figure 1. The circuit consists of two capacitors, two inductors, and one memristor. The memristor is characterized by its incremental memductance function $W(\varphi)$ describing the flux-dependent rate of change of charge:

$$
W(\varphi(t))=\frac{d q(\varphi(t))}{d \varphi(t)}=-a+3 b \varphi^{2}(t)
$$

where $a>0, b>0$ are constants.
And the equations for the circuit are described by

$$
\begin{aligned}
& \frac{d v_{1}(t)}{d t}=\frac{1}{C_{1}}\left(i_{3}(t)-W(\varphi(t)) v_{1}(t)\right), \\
& \frac{d v_{2}(t)}{d t}=\frac{1}{C_{2}}\left(-i_{3}(t)+i_{4}(t)\right), \\
& \frac{d i_{3}(t)}{d t}=\frac{1}{L_{1}}\left(v_{2}(t)-v_{1}(t)-R i_{3}(t)\right), \\
& \frac{d i_{4}(t)}{d t}=\frac{-v_{2}(t)}{L_{2}}, \\
& \frac{d \varphi(t)}{d t}=v_{1}(t),
\end{aligned}
$$

where $W(\varphi(t))=-a+3 b \varphi^{2}(t)$.

Letting $x_{1}(t)=v_{1}(t), x_{2}(t)=v_{2}(t), x_{3}(t)=i_{3}(t), x_{4}(t)=$ $i_{4}(t)$, and $x_{5}(t)=\varphi(t)$, system (2) can be further rewritten as

$$
\begin{aligned}
& \frac{d x_{1}(t)}{d t}=\frac{1}{C_{1}}\left(x_{3}(t)-W\left(x_{5}(t)\right) x_{1}(t)\right), \\
& \frac{d x_{2}(t)}{d t}=\frac{1}{C_{2}}\left(-x_{3}(t)+x_{4}(t)\right) \\
& \frac{d x_{3}(t)}{d t}=\frac{1}{L_{1}}\left(x_{2}(t)-x_{1}(t)-R x_{3}(t)\right), \\
& \frac{d x_{4}(t)}{d t}=\frac{-x_{2}(t)}{L_{2}} \\
& \frac{d x_{5}(t)}{d t}=x_{1}(t)
\end{aligned}
$$

When $1 / C_{1}=9, C_{2}=1,1 / L_{1}=30,1 / L_{2}=15, R=$ $1, a=1.2$, and $b=0.4$, system (2) exhibits chaos or hyperchaos as shown in Figure 2.

\section{Synchronization of Hyperchaotic Systems- Based Memristor}

Let system (3) be the drive system. In what follows, the coupled response system with feedback control is given by

$$
\begin{aligned}
& \frac{d y_{1}(t)}{d t}=\frac{1}{C_{1}}\left(y_{3}(t)-W\left(y_{5}(t)\right) y_{1}(t)\right)+u_{1}(t), \\
& \frac{d y_{2}(t)}{d t}=\frac{1}{C_{2}}\left(-y_{3}(t)+y_{4}(t)\right)+u_{2}(t), \\
& \frac{d y_{3}(t)}{d t}=\frac{1}{L_{1}}\left(y_{2}(t)-y_{1}(t)-R y_{3}(t)\right)+u_{3}(t), \\
& \frac{d y_{4}(t)}{d t}=\frac{-y_{2}(t)}{L_{2}}+u_{4}(t), \\
& \frac{d y_{5}(t)}{d t}=y_{1}(t)+u_{5}(t),
\end{aligned}
$$




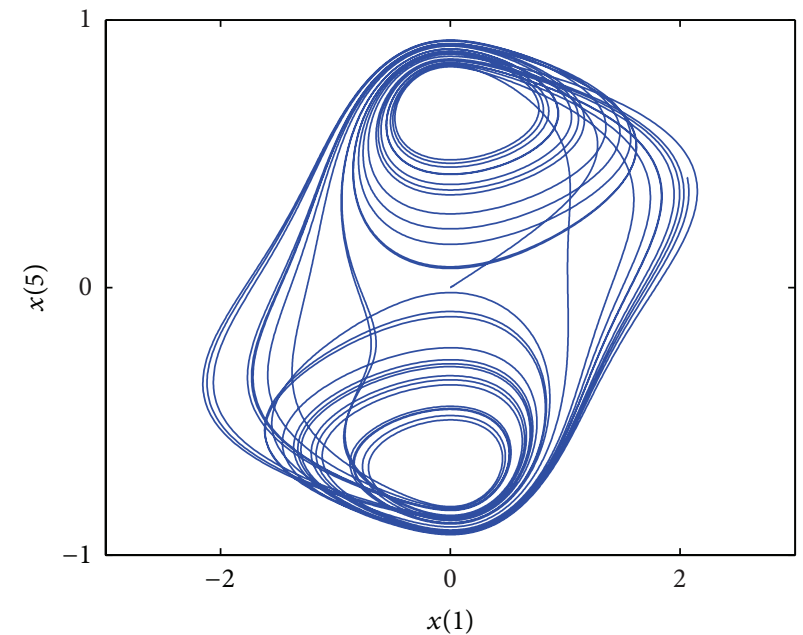

(a)

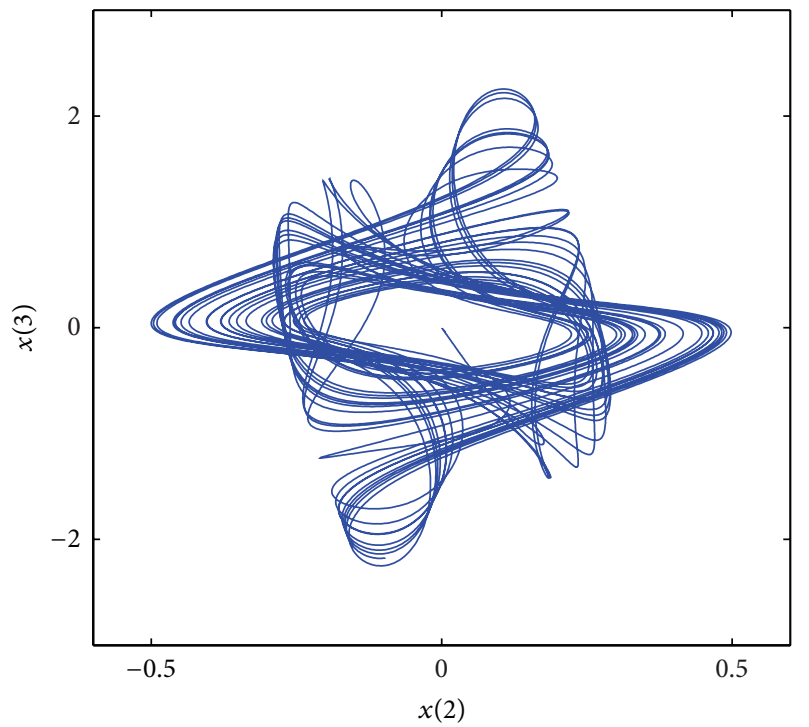

(c)

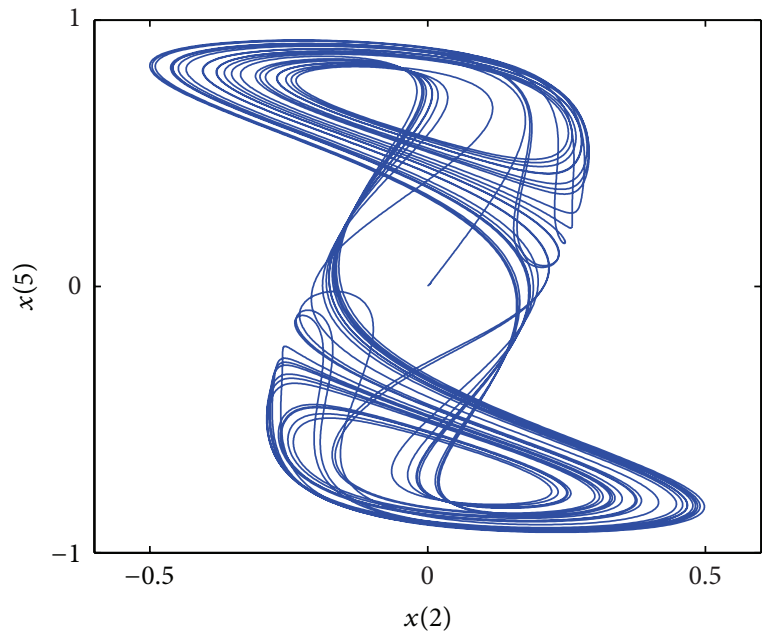

(b)

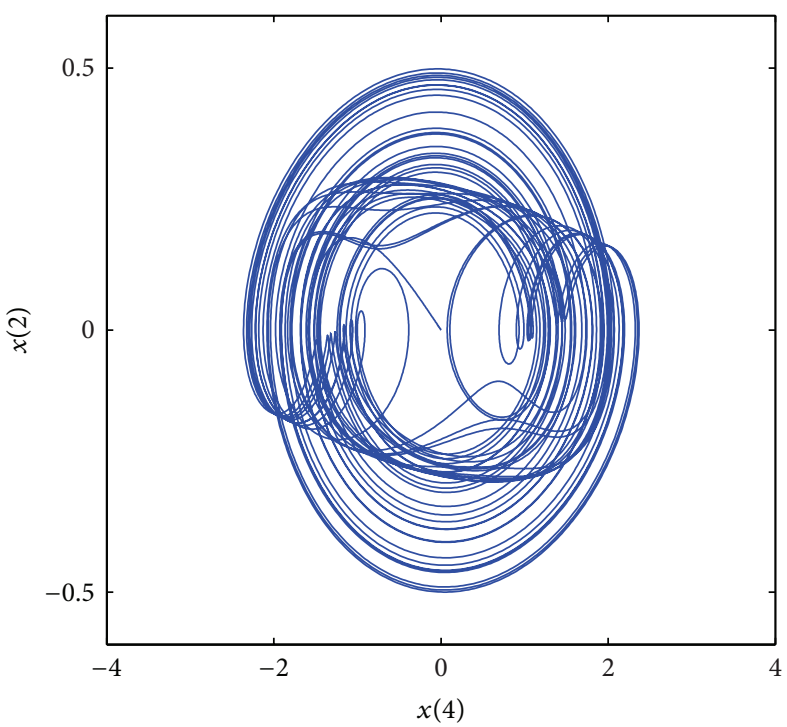

(d)

FIgURE 2: The chaotic attractor of the memristor oscillator.

where $u_{1}(t), u_{2}(t), u_{3}(t), u_{4}(t), u_{5}(t)$ are the control function defined by

$$
\begin{aligned}
u_{1}(t)=k_{1} & \left(y_{1}(t)-x_{1}(t)\right)+\frac{1}{C_{1}}\left(3 b y_{5}^{2}(t) y_{1}(t)\right) \\
- & \frac{1}{C_{1}}\left(3 b x_{5}^{2}(t) x_{1}(t)\right), \\
u_{2}(t) & =k_{2}\left(y_{2}(t)-x_{2}(t)\right), \\
u_{3}(t) & =k_{3}\left(y_{3}(t)-x_{3}(t)\right), \\
u_{4}(t) & =k_{4}\left(y_{4}(t)-x_{4}(t)\right), \\
u_{5}(t) & =k_{5}\left(y_{5}(t)-x_{5}(t)\right) .
\end{aligned}
$$

Letting $e_{i}(t)=y_{i}(t)-x_{i}(t), i=1,2,3,4,5$, be the synchronization error between systems (3) and (4) yields the error system

$$
\begin{gathered}
\dot{e}_{1}(t)=\frac{1}{C_{1}}\left(e_{3}(t)+a e_{1}(t)\right)+k_{1} e_{1}(t), \\
\dot{e}_{2}(t)=\frac{1}{C_{2}}\left(-e_{3}(t)+e_{4}(t)\right)+k_{2} e_{2}(t), \\
\dot{e}_{3}(t)=\frac{1}{L_{1}}\left(e_{2}(t)-e_{1}(t)-R e_{3}(t)\right)+k_{3} e_{3}(t), \\
\dot{e}_{4}(t)=\frac{-e_{2}(t)}{L_{2}}+k_{4} e_{4}(t) \\
\dot{e}_{5}(t)=e_{1}(t)+k_{5} e_{5}(t)
\end{gathered}
$$

We now state our main results. 
Theorem 1. Suppose that there exist positive constants $k_{1}, k_{2}$, $k_{3}, k_{4}, k_{5}$, such that

$$
P=\left[\begin{array}{ccccc}
k_{1}+\frac{a}{C_{1}} & 0 & \frac{1}{2 C_{1}}-\frac{1}{2 L_{1}} & 0 & \frac{1}{2} \\
0 & k_{2} & -\frac{1}{2 C_{2}}+\frac{1}{2 L_{1}} & \frac{1}{2 C_{2}}-\frac{1}{2 L_{2}} & 0 \\
\frac{1}{2 C_{1}}-\frac{1}{2 L_{1}} & -\frac{1}{2 C_{2}}+\frac{1}{2 L_{1}} & -\frac{R}{L_{1}}+k_{3} & 0 & 0 \\
0 & \frac{1}{2 C_{2}}-\frac{1}{2 L_{2}} & 0 & k_{4} & 0 \\
\frac{1}{2} & 0 & 0 & 0 & k_{5}
\end{array}\right] \leq 0
$$

then the synchronization error system (6) is asymptotically stable, and the systems (3) and (4) are asymptotically synchronized.

Proof. Choose the Lyapunov function as follows:

$$
V(t)=\frac{1}{2}\left(e_{1}(t)^{2}+e_{2}(t)^{2}+e_{3}(t)^{2}+e_{4}(t)^{2}+e_{5}(t)^{2}\right) .
$$
(6) is

Then the differentiation of $V$ along trajectories of system

$$
\begin{aligned}
& \dot{V}(t) \\
& =e_{1}(t)\left(\frac{1}{C_{1}}\left(e_{3}(t)+a e_{1}(t)\right)+k_{1} e_{1}(t)\right) \\
& \quad+e_{2}(t)\left(\frac{1}{C_{2}}\left(-e_{3}(t)+e_{4}(t)\right)+k_{2} e_{2}(t)\right) \\
& \quad+e_{3}(t)\left(\frac{1}{L_{1}}\left(e_{2}(t)-e_{1}(t)-\operatorname{Re}_{3}(t)\right)+k_{3} e_{3}(t)\right) \\
& \quad+e_{4}(t)\left(\frac{-e_{2}(t)}{L_{2}}+k_{4} e_{4}(t)\right)
\end{aligned}
$$

where $e(t)=\left(e_{1}(t), e_{2}(t), e_{3}(t), e_{4}(t), e_{5}(t)\right)^{T}$, and

$$
P=\left[\begin{array}{ccccc}
k_{1}+\frac{a}{C_{1}} & 0 & \frac{1}{2 C_{1}}-\frac{1}{2 L_{1}} & 0 & \frac{1}{2} \\
0 & k_{2} & -\frac{1}{2 C_{2}}+\frac{1}{2 L_{1}} \frac{1}{2 C_{2}}-\frac{1}{2 L_{2}} & 0 \\
\frac{1}{2 C_{1}}-\frac{1}{2 L_{1}} & -\frac{1}{2 C_{2}}+\frac{1}{2 L_{1}} & -\frac{R}{L_{1}}+k_{3} & 0 & 0 \\
0 & \frac{1}{2 C_{2}}-\frac{1}{2 L_{2}} & 0 & k_{4} & 0 \\
\frac{1}{2} & 0 & 0 & 0 & k_{5}
\end{array}\right] .
$$




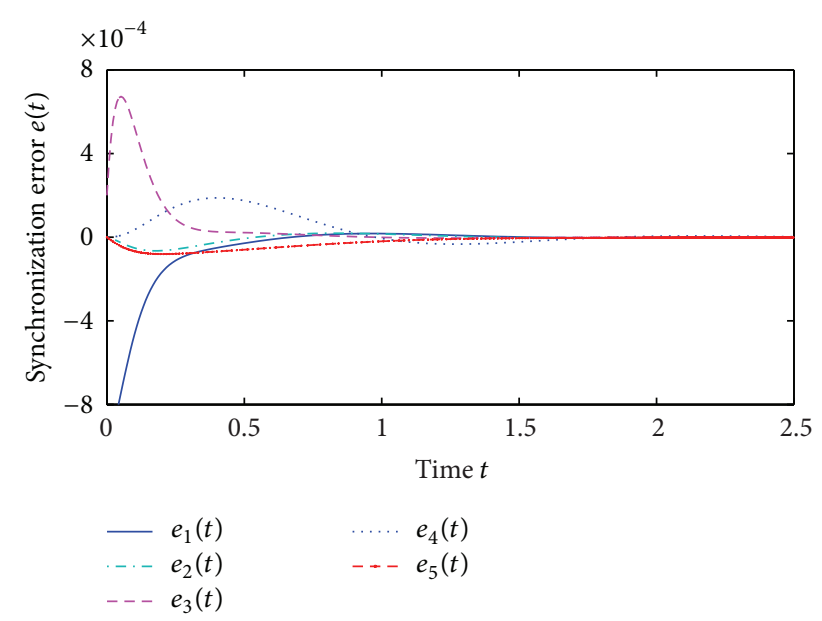

FIGURE 3: Synchronization error of memristor-based hyperchaotic systems.

According to Lyapunov theory, the inequality $\dot{V}(t) \leq 0$ indicates $V(t)$ converges to zero asymptotically; that is, the error system $e(t)$ converges to zero globally and asymptotically, and the synchronization between system (3) and system (4) is achieved.

In simulation, we select the parameters of memristorbased Chua's system as initial values of drive and response systems are $\left(x_{1}(0), x_{2}(0), x_{3}(0), x_{4}(0), x_{5}(0)\right)=$ $(0.001,0,-0.0001,0,0)$, and $\quad\left(y_{1}(0), y_{2}(0), y_{3}(0), y_{4}(0)\right.$, $\left.y_{5}(0)\right)=(0,0,0.0001,0,0)$, respectively. From Theorem 1, the control gain is chosen as $k_{1}=-11, k_{2}=k_{3}=k_{4}=k_{5}=-2$. We plot the time response curves of the synchronization error system as shown in Figure 3. From Figure 3, one can see that the synchronization error of the hyperchaotic systems is asymptotically stable; that is, the synchronization between systems (3) and (4) is achieved.

\section{Lag Synchronization of Hyperchaotic Systems}

In this section, we study the lag synchronization of memristor-based Chua's circuit. The system (3) can be rewritten with two parts as follows:

$$
\dot{x}(t)=A x(t)+B f(x(t)),
$$

where $x(t)=\left(x_{1}(t), x_{2}(t), x_{3}(t), x_{4}(t), x_{5}(t)\right)^{T}$,

$$
A=\left(\begin{array}{ccccc}
a & 0 & \frac{1}{C_{1}} & 0 & 0 \\
0 & 0 & -\frac{1}{C_{2}} & \frac{1}{C_{2}} & 0 \\
-\frac{1}{L_{1}} & \frac{1}{L_{1}} & -\frac{R}{L_{1}} & 0 & 0 \\
0 & \frac{-1}{L_{2}} & 0 & 0 & 0 \\
1 & 0 & 0 & 0 & 0
\end{array}\right),
$$

$$
\begin{gathered}
f(x(t))=\left(\begin{array}{c}
3 b x_{5}^{2} x_{1} \\
0 \\
0 \\
0 \\
0
\end{array}\right), \\
B=\left(\begin{array}{ccccc}
-\frac{1}{C_{1}} & 0 & 0 & 0 & 0 \\
0 & 0 & 0 & 0 & 0 \\
0 & 0 & 0 & 0 & 0 \\
0 & 0 & 0 & 0 & 0 \\
0 & 0 & 0 & 0 & 0
\end{array}\right) .
\end{gathered}
$$

As for vector function $f(x)$, assume that for any $x, y \in \Omega$ we have

$$
\left|f_{i}(x)-f_{i}(y)\right| \leq L_{\max }|x-y|, \quad i=1,2,3,4,5 .
$$

The above condition is considered as the uniform Lipschitz condition, and $L_{\max }>0$ refers to the uniform Lipschitz constant.

We construct the response system as follows:

$$
\dot{y}(t)=A y(t)+B f(y(t))+u(t),
$$

where $y(t)=\left(y_{1}(t), y_{2}(t), y_{3}(t), y_{4}(t), y_{5}(t)\right)^{T}$ is the response state and $u(t)$ is the control gain defined by

$$
u(t)=k(y(t)-x(t-\tau)),
$$

where $\tau>0$ is the propagation delay and $k$ denotes control strength. Let $e(t)=y(t)-x(t-\tau)$ be the lag synchronization error between the systems (11) and (14); then the error system is

$$
\begin{aligned}
\dot{e}(t) & =\dot{y}(t)-\dot{x}(t-\tau) \\
& =A e(t)+B(f(y)-f(x(t-\tau)))+u(t) \\
& =A e(t)+B(f(y)-f(x(t-\tau)))+k e(t) .
\end{aligned}
$$

We now state our main results.

Theorem 2. Suppose that there exist positive constants $s_{1}$ and $g_{1}$ such that

$$
A+A^{T}+2 k I+s_{1}\left(B+B^{T}\right)+s_{1}^{-1} L_{\max }^{2} I+g_{1} I \leq 0 .
$$

Then, the synchronization error system (16) is globally exponentially stable, and the systems (11) and (14) are globally exponentially lag-synchronized.

Proof. Choose the Lyapunov function as follows:

$$
V(t)=e(t)^{T} e(t) .
$$


Then the differentiation of $V$ along trajectories of (16) is $\dot{V}(t)$

$$
\begin{aligned}
= & e(t)^{T} \dot{e}(t)+\dot{e}(t)^{T} e(t) \\
= & e(t)^{T}[A e(t)+B(f(y)-f(x(t-\tau)))+k e(t)] \\
& +[A e(t)+B(f(y)-f(x(t-\tau)))+k e(t)]^{T} e(t) \\
\leq & e(t)^{T}\left[A+A^{T}+2 k I\right] e(t)+s_{1} e(t)^{T}\left[B+B^{T}\right] e(t) \\
& +s_{1}{ }^{-1}[f(y)-f(x(t-\tau))]^{T}[f(y)-f(x(t-\tau))] \\
\leq & e(t)^{T}\left[A+A^{T}+2 k I+s_{1}\left(B+B^{T}\right)\right] e(t) \\
& +s_{1}{ }^{-1} L_{\max }{ }^{2} e(t)^{T} e(t) \\
= & e(t)^{T}\left[A+A^{T}+2 k I+s_{1}\left(B+B^{T}\right)+s_{1}{ }^{-1} L_{\max }{ }^{2} I+g_{1} I\right] e(t) \\
& -g_{1} e(t)^{T} e(t) \\
\leq & -g_{1} e(t)^{T} e(t) \\
= & -g_{1} V(t) .
\end{aligned}
$$

According to Lyapunov theory, the inequality $\dot{V}(t) \leq$ $-g_{1} V(t)$ indicates $V(t)$ converges to zero exponentially. Furthermore, we can conclude that the lag synchronization error system $e(t)$ converges to zero globally and exponentially with exponential convergence rate $g_{1}$, and the lag synchronization between system (11) and system (14) can be obtained. This completes the proof.

Let $s_{1}=1$; one obtains from Theorem 2 the following corollary.

Corollary 3. If there exist positive constants $g_{1}$ such that

$$
k \leq \frac{\left(-\lambda_{\min }\left(A+A^{T}\right)-\lambda_{\min }\left(B+B^{T}\right)-L_{\max }^{2}-g_{1}\right)}{2},
$$

then the synchronization error system (16) is globally exponentially stable, and the systems (11) and (14) are globally exponentially lag-synchronized.

In simulation, we select the parameters of memristorbased Chua's system as initial values of drive and response systems are

$$
\begin{gathered}
\left(x_{1}(0), x_{2}(0), x_{3}(0), x_{4}(0), x_{5}(0)\right) \\
=(0.001,0,-0.0001,0,0), \\
\left(y_{1}(0), y_{2}(0), y_{3}(0), y_{4}(0), y_{5}(0)\right)=(0,0,0.0001,0,0) .
\end{gathered}
$$

Based on the bound of the hyperchaotic attractor, we can choose $L_{\max }=4.40832$. Respectively, from Corollary 3, we select $g_{1}=32, k=-1.1827$ and plot the lag synchronization

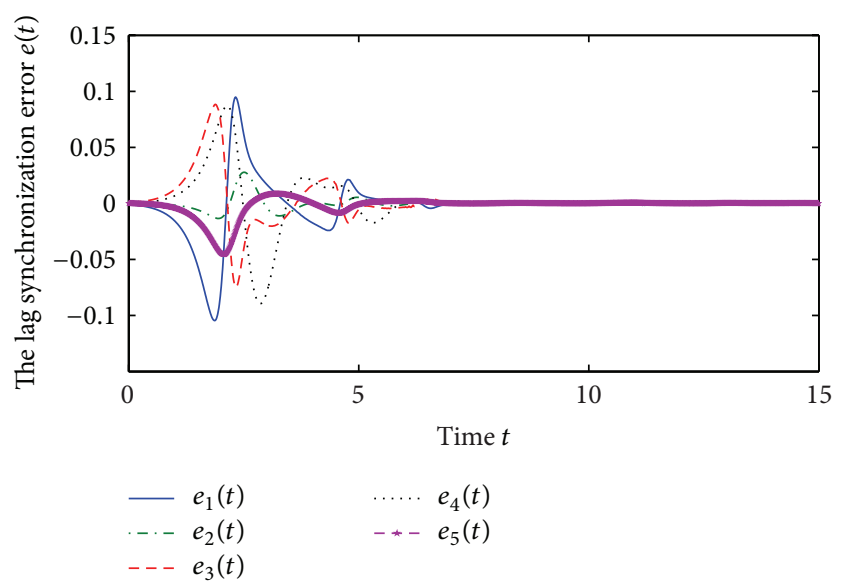

FIGURE 4: Lag synchronization error of memristor-based hyperchaotic systems.

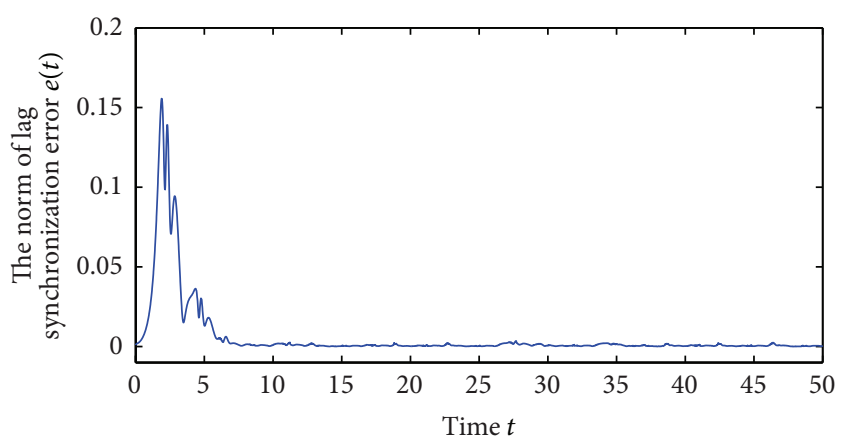

FIGURE 5: The norm of lag synchronization error of memristorbased hyperchaotic systems.

errors curve, as shown in Figure 4. Figure 5 shows the norm of lag synchronization error of the memristor-based hyperchaotic systems. As the time $t$ goes to infinity, the lag synchronization error system is exponentially stable. Hence, the lag synchronization between system (11) and system (14) is achieved.

\section{Conclusions}

This paper has studied the synchronization and lag synchronization of memristor-based 5D hyperchaotic circuits. The feedback controllers have been designed to stabilize the synchronization error system and lag synchronization error system. Simulation results were given to verify the effectiveness and feasibility of method.

\section{Conflict of Interests}

The authors declare that there is no conflict of interests regarding the publication of this paper. 


\section{Acknowledgments}

This publication was made possible by NPRP Grant no. NPRP-4-1162-181 from the Qatar National Research Fund (a member of Qatar Foundation). The statements made herein are solely the responsibility of the authors. This work was also supported by Natural Science Foundation of China (Grant no. 61374078, Grant no. 61403050, and Grant no. 61302180).

\section{References}

[1] L. O. Chua, "Memristor-the missing circuit element," IEEE Transactions on Circuit Theory, vol. 18, no. 5, pp. 507-519, 1971.

[2] D. B. Strukov, G. S. Snider, D. R. Stewart, and R. S. Williams, "The missing memristor found," Nature, vol. 453, no. 7191, pp. 80-83, 2008.

[3] Y. Ho, G. M. Huang, and P. Li, "Nonvolatile memristor memory: device characteristics and design implications," in Proceedings of the IEEE/ACM International Conference on Computer-Aided Design: Digest of Technical Papers (ICCAD '09), pp. 485-490, San Jose, Calif, USA, November 2009.

[4] J. Borghetti, G. S. Snider, P. J. Kuekes, J. J. Yang, D. R. Stewart, and R.S. Williams, "Memristive' switches enable "stateful" logic operations via material implication," Nature, vol. 464, no. 7290, pp. 873-876, 2010.

[5] T. Raja and S. Mourad, "Digital logic implementation in memristor-based crossbars: a tutorial," in Proceedings of the 5th IEEE International Symposium on Electronic Design, Test and Applications (DELTA '10), pp. 303-309, January 2010.

[6] S. H. Jo, T. Chang, I. Ebong, B. B. Bhadviya, P. Mazumder, and W. Lu, "Nanoscale memristor device as synapse in neuromorphic systems," Nano Letters, vol. 10, no. 4, pp. 1297-1301, 2010.

[7] Y. V. Pershin, S. L. Fontaine, and M. D. Ventra, "Memresistive model of amoeba's learning," Physical Review E, vol. 80, Article ID 021926, 2009.

[8] Y. V. Pershin and M. Di Ventra, "Experimental demonstration of associative memory with memristive neural networks," Neural Networks, vol. 23, no. 7, pp. 881-886, 2010.

[9] A. Afifi, A. Ayatollahi, and F. Raissi, "Implementation of biologically plausible spiking neural network models on the memristor crossbar-based CMOS/nano circuits," in Proceedings of the European Conference on Circuit Theory and Design (ECCTD '09), pp. 563-566, Antalya, Turkey, 2009.

[10] M. Itoh and L. O. Chua, "Memristor oscillators," International Journal of Bifurcation and Chaos, vol. 18, no. 11, pp. 3183-3206, 2008.

[11] B. Muthuswamy and P. P. Kokate, "Memristor based chaotic circuits," IETE Technical Review, vol. 26, no. 6, pp. 415-426, 2009.

[12] B.-C. Bao, J.-P. Xu, and Z. Liu, "Initial state dependent dynamical behaviors in a memristor based chaotic circuit," Chinese Physics Letters, vol. 27, no. 7, Article ID 070504, 2010.

[13] B. C. Bao, Q. H. Wang, and J. P. Xu, "On memristor based fiveorder chaotic circuit," Journal of Circuits and Systems, vol. 16, no. 2, p. 66, 2011.

[14] A. L. Fitch, D. S. Yu, H. H. C. Iu, and V. Sreeram, "Hyperchaos in a memeristor-based modified canonical chuas circuit," International Journal of Bifurcation and Chaos, vol. 22, no. 6, Article ID 1250133, 2012.

[15] G. D. Zhang, Y. Shen, Q. Yin, and J. W. Sun, "Global exponential periodicity and stability of a class of memristor-based recurrent neural networks with multiple delays," Information Sciences, vol. 232, pp. 386-396, 2013.

[16] J. Sun, Y. Shen, Q. Yin, and C. Xu, “Compound synchronization of four memristor chaotic oscillator systems and secure communication," Chaos, vol. 23, no. 1, Article ID 013140, 2013.

[17] S. P. Wen, Z. G. Zeng, and T. W. Huang, "Adaptive synchronization of memristor-based Chua's circuits," Physics Letters Section A, vol. 376, no. 44, pp. 2775-2780, 2012.

[18] A. L. Wu, S. P. Wen, and Z. G. Zeng, "Synchronization control of a class of memristor-based recurrent neural networks," Information Sciences, vol. 183, no. 1, pp. 106-116, 2012.

[19] J. Huang, C. Li, and X. He, "Stabilization of a memristor-based chaotic system by intermittent control and fuzzy processing," International Journal of Control, Automation and Systems, vol. 11, no. 3, pp. 643-647, 2013.

[20] G. Grassi and S. Mascolo, "A system theory approach for designing cryptosystems based on hyperchaos," IEEE Transactions on Circuits and Systems I: Fundamental Theory and Applications, vol. 46, no. 9, pp. 1135-1138, 1999.

[21] R. Jia, Q. Huang, and J. Peng, "Study of the hyperchaos-based hash function in e-commerce applications," in Proceedings of the International Conference on Intelligent Computation Technology and Automation (ICICTA '10), pp. 451-454, May 2010. 


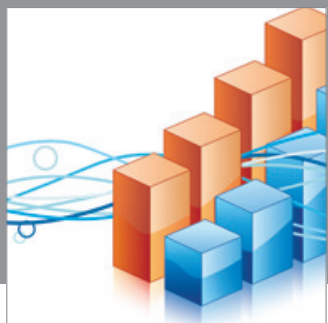

Advances in

Operations Research

mansans

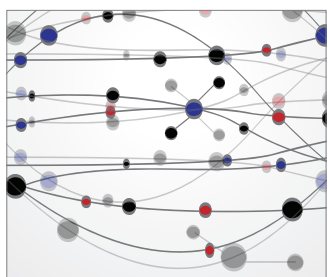

The Scientific World Journal
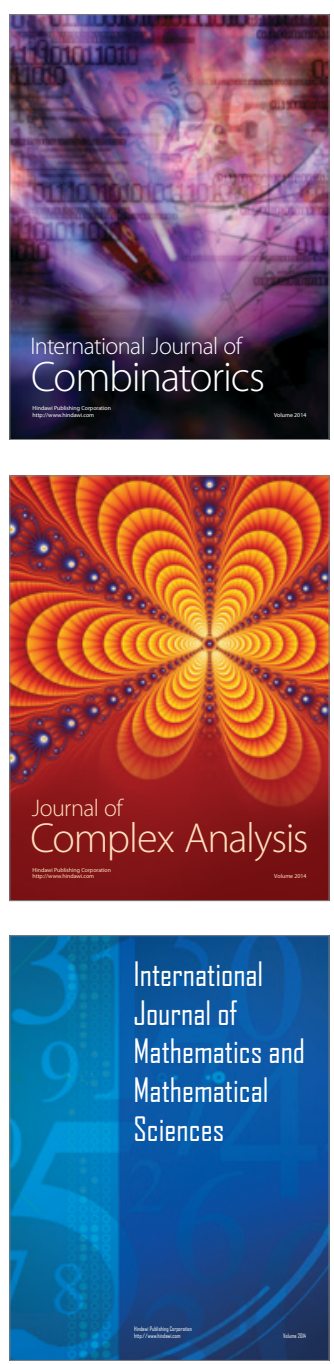
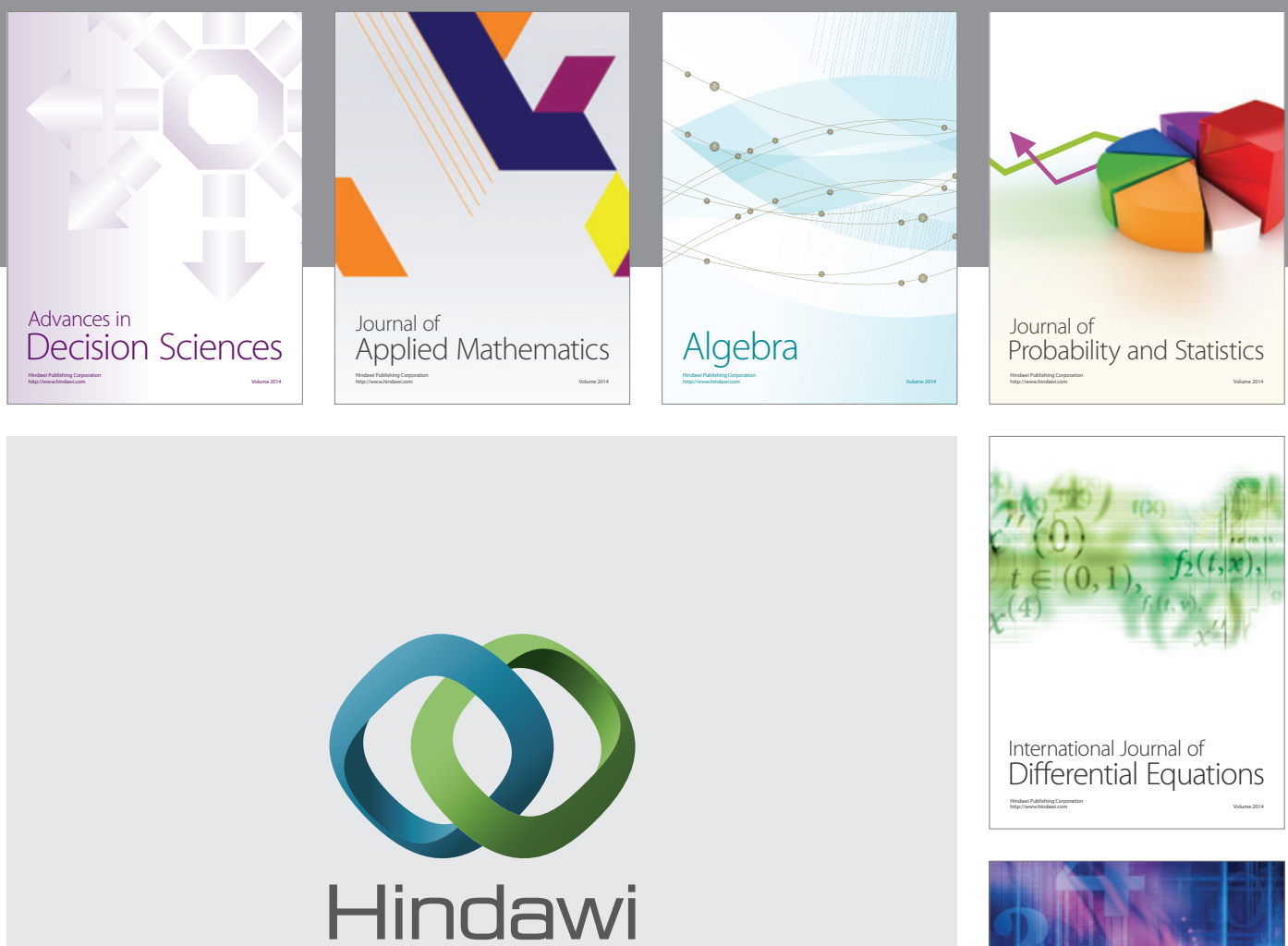

Submit your manuscripts at http://www.hindawi.com
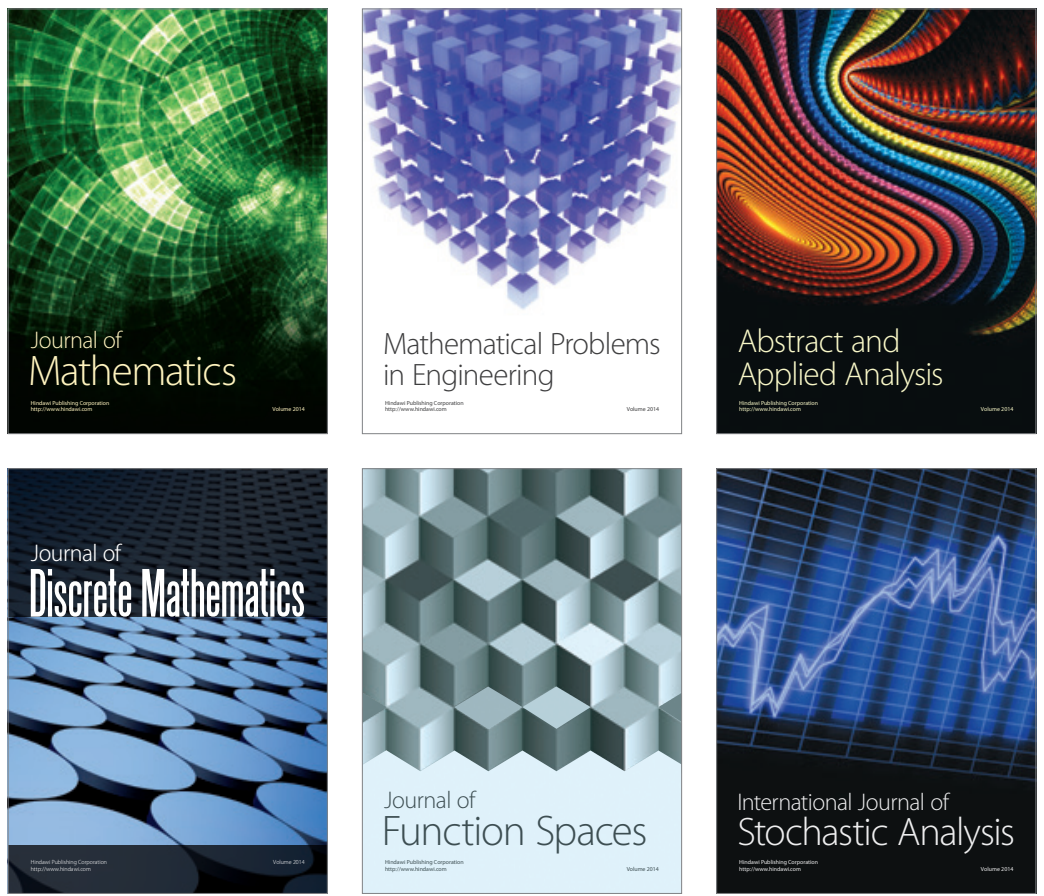

Journal of

Function Spaces

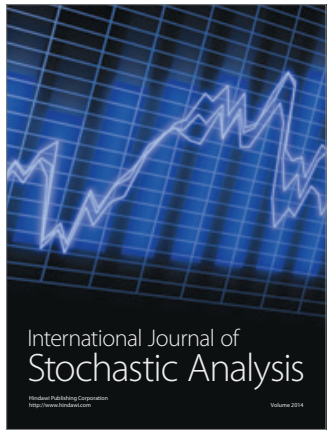

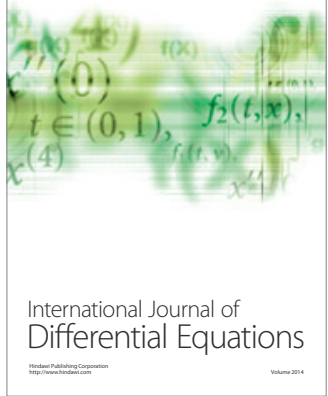
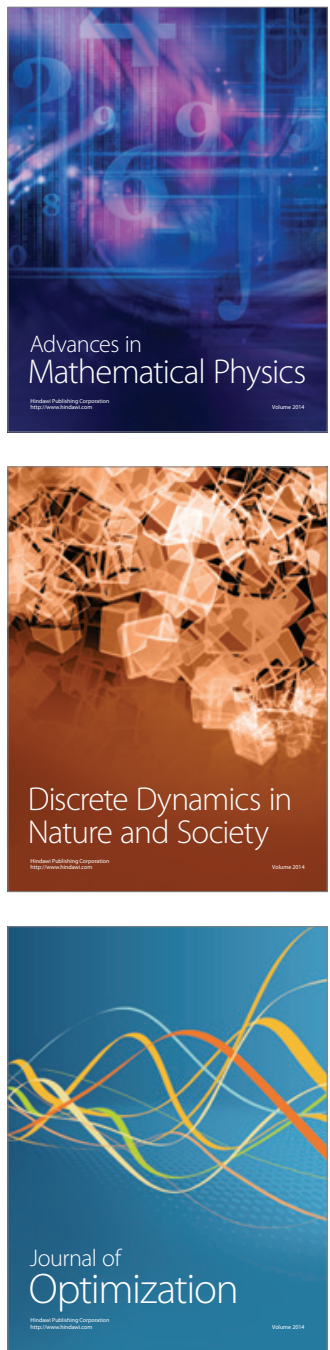